\title{
LETTERS
}

\section{Underlying maternal infection likely cause of study findings}

We would like to contextualize this CMAJ article $^{1}$ in the larger role of infection and antibiotics in pregnancy and pregnancy outcomes. Antibiotics are commonly prescribed in pregnancy, and careful consideration should always be taken with regards to the appropriate indication and choice of antibiotic therapy. This article states that prescription of macrolides (excluding erythromycin), quinolones, tetracyclines, sulfonamides and metronidazole during early pregnancy resulted in higher rates of spontaneous miscarriage.

However, this study has multiple limitations, which are underemphasized. The foremost is the role of infection itself being associated with increased risk of spontaneous loss, as well as harm to maternal health. Given the breadth of antibiotics from multiple classes implicated in the increased risk of loss, it is highly likely that the underlying maternal infection, and not the antibiotic prescription itself, is the source of increased spontaneous loss.

This study in its current design and analysis cannot properly address the role of serious, complex infections in the risk of spontaneous abortion. We agree with the authors that the presence of unmeasured confounders contributing to the above findings is very plausible.
It is important to emphasize that any infection during pregnancy poses risk to both the developing fetus and the mother, and should be considered as potentially high risk owing to the underlying nature of pregnancy.

Therefore, we encourage care providers to prescribe appropriate antibiotic therapy during pregnancy in any situation where there is evidence of bacterial infection. The safest environment for a developing fetus is a healthy mother.

\section{Chelsea Elwood MD}

Reproductive Infectious Diseases Fellow

\section{Deborah Money MD}

Professor, Division of Gynaecologic Specialities, Department of Obstetrics and Gynaecology, Faculty of Medicine, The University of British Columbia, Vancouver, BC Members, Society of Obstetricians and Gynaecologists (SOGC) Infectious

Diseases Committee

- Cite as: CMAJ 2017 July 10;189:E918. doi: $10.1503 / \mathrm{cmaj} .733199$

\section{References}

1. Muanda FT, Sheehy O, Bérard A. Use of antibiotics during pregnancy and risk of spontaneous abortion. CMAJ 2017;189:E625-33.

Competing interests: None declared. 